\title{
ExTra: Expediting File Transfers through Optimized Inter-vehicle Communication
}

\author{
Panayiotis Kolios, Christos Panayiotou and Georgios Ellinas
}

\begin{abstract}
In the realm of cooperative Intelligent Transportation Systems, vehicles are able to communicate with each other and with the available telecommunications infrastructure to support various safety-related services, traffic-efficiency solutions, and a wide variety of infotainment applications. As the number of mobile devices and information they need to exchange steadily increases, this will further strain mobile networks. It is for that reason that communication over wireless local area networks (WLANs) is becoming the primary means of transporting data to/from vehicles.

However, due to their limit range WLANs only offer intermittent connectivity, and due to node mobility the established connections are only available for a short period of time. The present paper investigates how inter-vehicle communication can help minimize the upload/download delivery times in the context of delay tolerant traffic and intermittent communication. To do so, each vehicular station distributes part of its message to neighboring nodes prior to accessing the infrastructure. In turn, vehicles transmit their buffered messages when passing by the infrastructure terminals to complete the file transfer. This paper presents a thorough study of the aforementioned strategy providing a mathematical framework and optimized forwarding techniques. Performance results validate the applicability of the proposed solution.
\end{abstract}

Index Terms-Cellular Networks, Vehicle-to-Vehicle Communications, Data Offloading, Networked Computation and Control

\section{INTRODUCTION}

Judging from the heightened level of understanding for vehicular communications attained by the research community [1], and the maturity being reached by field operational tests currently undertaken by industrial partners [2], it becomes apparent that vehicular technologies are set for prime time.

Nevertheless, while the complete communication protocol suite is in place for both vehicle-to-vehicle (V2V) and vehicleto-infrastructure (V2I) communication, the networking aspects are far from being finalized [3]. This is partly due to the fact that vehicular networking is envisioned to support a broad range of solutions, including road safety, traffic efficiency, and infotainment services [4], all of which necessitate different requirements that need to be carefully designed and standardized [5]. An even bigger problem however (that relates more to the technical considerations) is the fact that the introduction of large volumes of vehicular stations (VSs) and the associated data traffic generated by these devices can easily overconsume the available capacity of today's mobile networks.

P. Kolios, C. Panayiotou, and G. Ellinas are members of the KIOS Research Center for Intelligent Systems and Networks and the Department of Electrical and Computer Engineering, University of Cyprus, e-mail:\{pkolios, christosp, gellinas\}@ucy.ac.cy
Already, mobile operators struggle to support the increasing number of smartphone users that access the Internet through the available cellular infrastructure [7]. To exacerbate this problem further, vehicles (currently exceeding the 1 billion mark and projected to go beyond 3 billion units worldwide by 2050 [6]) can be fitted with powerful communication devices that are less confined by space and less constrained by energy resources. In that respect, onboard units can continuously monitor and log sensory readings, capture multimedia content, and maintain social interactions, all of which accumulate to large volumes of data that inevitably need to be forwarded to the fixed network.

However, the potential of deploying additional cellular infrastructure to support higher traffic has long been ruled out by mobile operators due to the high capital expenditure and the marginal returns anticipated by the investment. On the contrary, mobile operators have been keen to the idea of offloading Internet traffic over the available WLANs (e.g., WiFi or femtocells) and this idea has prevailed ever since the maturity of the smartphone market. Indicatively, Cisco identified that of the total mobile data traffic, $11 \%$ was offloaded to fixed/WiFi networks in year 2011 while the projected figure is set to double by 2016 [8].

Thus, new architectures [9] and techniques [10] are currently being researched for, in order to support vehicular data traffic offloading. In line with these efforts, the work documented hereafter is concerned with offloading data through WLAN access points (APs). However, unlike previous studies that are solely focused on the performance of the transmission link established between VSs and APs, this work takes on the task of maximizing transmission opportunities by capitalizing on inter-vehicle communication prior to contacting the infrastructure. Arguably, a link between a VS node and an AP could be short-lived due to mobility of the VS and the limited coverage of the AP [11]. On the other hand, a group of vehicles could maintain inter-vehicle communication for extended periods of time while collectively these vehicles can have a multiplicity of transmission opportunities to the infrastructure.

For illustration purposes consider the communication paradigm depicted in Figure 1, where a group of three VSs follows a journey along a specific roadway. Within this group, let us assume vehicle $a$ intends to upload a file to the infrastructure via the available AP. To ensure that the complete file is successfully transmitted to the infrastructure, vehicle $a$ sends out part of its file to both vehicles $b$ and $c$, as shown by point 1 in the figure. Subsequently, each vehicle forwards its part of the file to the infrastructure when it passes by the AP, 
as shown by point 2 in the figure.

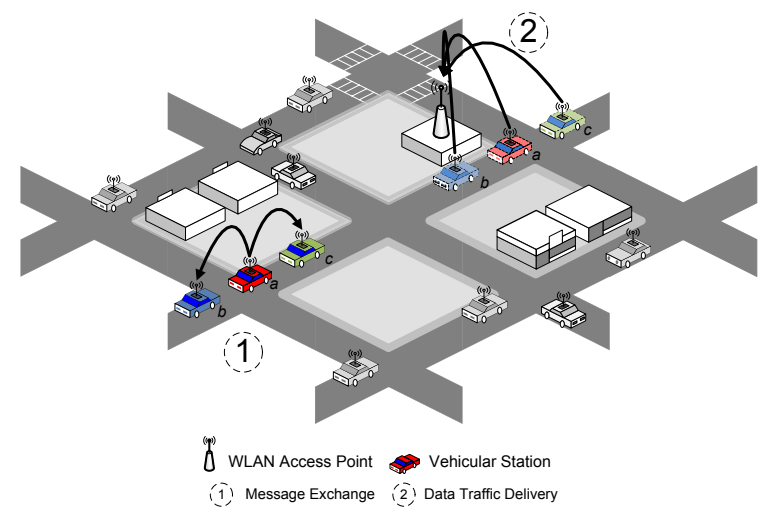

Fig. 1. Communication paradigm under investigation includes the exchange of file segments between cooperating vehicles before transiting to the infrastructure.

Evidently, a file that is large enough to require a transmission time greater than the link duration will benefit from the proposed strategy since in the alternative case a vehicle would need to withhold the message for future communication opportunities. Smaller-size files can also benefit from the proposed strategy as well, since minimizing the amount of data that needs to be transmitted by each vehicle at any one time allows for increasing retransmission opportunities that might be necessary when communication errors occur. Overall, the proposed strategy offers the potential to expedite the exchange of files between vehicles and the infrastructure by distributing file segments to adjacent nodes prior to communication with the infrastructure. The aforementioned strategy is referred to hereafter as ExTra. The ultimate goal of ExTra is to expedite delivery by evenly distributing data files across adjacent vehicles that in turn deliver these files to the infrastructure. Importantly, this task becomes viable due to the requirement for vehicles to broadcast location and mobility related information to other stations in their vicinity [12], revealing the instantaneous network topology.

In the rest of the paper, a systematic approach is followed in designing and modelling ExTra. More specifically, Section II describes the communications model under investigation and formulates a mathematical programming problem that optimizes the exchange of files between vehicles. Section III presents experimental results for the proposed solution, quantifies and analyzes the performance of the proposed solution. Section IV discusses practical considerations and investigates extensions of ExTra. Finally, Section V concludes the paper with the key findings of the documented work.

\section{NETWORKING MODEL}

\section{A. Problem Statement}

As discussed in the introduction, a particular vehicular station $a$ is considered that wants to forward its data to the infrastructure. To do so, it cooperates with adjacent stations and data files are exchanged between all neighboring stations to equally distribute the traffic across all neighbors. The ultimate goal is to successfully deliver data files at the earliest possible time. In order to realize this solution, the following are required:

R1 : VS $a$ creates a list of all stations from which it receives broadcasted messages indicating their identities, position, heading, and speed.

R2 : VS a receives information on the volume of data traffic already buffered at each station in the group of cooperating nodes that travel in the same direction.

Within cooperative ITS [12], each VS is required to periodically broadcast mobility related information including all parameters set in requirement R1 above. The information in R2 can be disseminated in a similar manner between collocated stations adding only minimal extra overhead to the broadcasted beacons.

With this information at hand, a station will be in a position to make informed decision on the reallocation of fragments between cooperating nodes; the subject matter of this work.

\section{B. Problem Formulation}

Consider a group of vehicles, as observed by a given source node. These vehicles, belonging to set $\mathcal{M}=\{1, \ldots, M\}$, travel along an arbitrary route in a road network. Let $\left(p_{m}, t\right), m \in \mathcal{M}$ be the geographical position $p_{m}$ of vehicle $m$ at time instance $t$ over the time horizon $\mathcal{T}=\{0, \ldots, T\}$. Hence, the current position of a vehicle is $\left(p_{m}, t_{1}\right)$ while its forecasted position one time instance ahead is $\left(p_{m}, t_{2}\right)$, and so forth. We assume that each of these vehicles has an onboard computing and communications device that can process and exchange data. Let $D$ be the communication range of each vehicular station (VS) and $R$ be the achievable data rate of the transmission circuitry. Moreover, each VS $m \in \mathcal{M}$ is assumed to be in possession of a file that consists of a total of $F_{m}$ packets and the aggregated load is simply $F=\sum_{m=1}^{M} F_{m}$. The transmission time for a single packet is $\tau$.

Using this information, a time-expanded graph is constructed which includes estimates of future vehicle positions at consecutive time instances of duration $\tau$. Effectively, this graph captures the path of vehicles over time. Moreover, the duration of consecutive replication epochs, on the graph, is set to $\tau$ units of time such that a single packet can be transmitted from one VS to another during a single time lapse. We create a space-time network graph $G=(\mathcal{V}, \mathcal{E})$ where $\mathcal{V}$ is the set of vertices and $\mathcal{E}$ is the set of edges. $\mathcal{V}=\left\{V_{p}, \mathrm{SS}\right\}$ consists of all VS replicas $V_{p}$ in time and space, and a super sink node (SS) that is used as a load aggregator for the collection of data files in the presented model. The set of edges $\mathcal{E}$ consists of the following three subsets:

$\mathcal{E}_{1}$ : Edges in $\mathcal{E}_{1}$ represent inter-vehicle transmission links.

$\mathcal{E}_{2}$ : Edges in $\mathcal{E}_{2}$ represent internal buffering of packets that takes place when a vehicle moves towards successive vehicle positions.

$\mathcal{E}_{3}$ : Edges in $\mathcal{E}_{3}$ represent virtual links that allow the flow of packets from each VS to SS, used to model the load imbalance cost.

Consider first an arbitrary link in $\mathcal{E}_{1}$ which indicates a transmission between two VSs. As indicated above, a packet 
exchange takes place over one time epoch and thus transmissions commencing at time instance $t$ terminate at time instance $t+1$. Hence, a transmission link between a pair of VSs is defined as follows, $(i \mapsto j) \in \mathcal{E}_{1}$ where $i=\left(p_{m}, t\right), m \in \mathcal{M}$ and $j=\left(p_{n}, t+1\right), n \neq m, n \in \mathcal{M}$. Moreover, a link in $\mathcal{E}_{2}$ dictates the buffering that takes place when a VS physically carries packets while on the move. As such, a buffer link is defined as $(i \mapsto j) \in \mathcal{E}_{2}$ where $i=\left(p_{m}, t\right), m \in \mathcal{M}$ and $j=\left(p_{m}, t+1\right), m \in \mathcal{M}$. Lastly, edges $(i \mapsto j)^{k} \in \mathcal{E}_{3}, i=$ $\left(p_{m}, T\right), j=\mathrm{SS}, k=1, \ldots, F$ connect the last set of VS replicas to SS. Notice that in this case multiple edges exists to reflect on the numerous packets that can be collected by each VS before engaging in transmission with the AP.

To aid understanding, Figure 2 illustrates one realization of the graph where three nodes travel from left to right on the plane. This time-space graph illustrates possible connections that could take place between vehicles. The networking

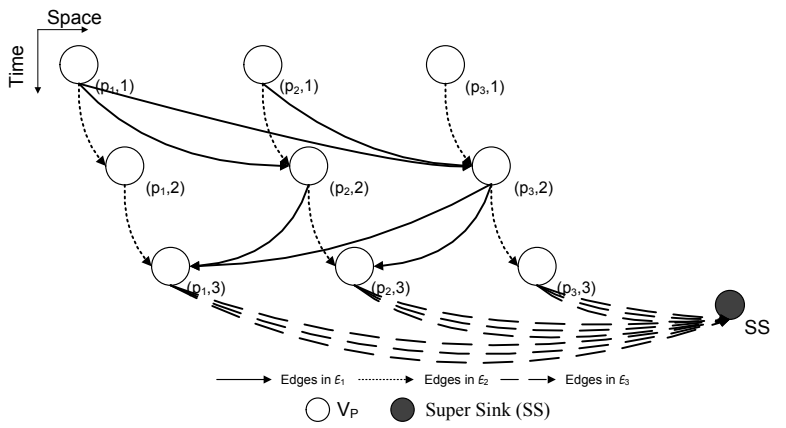

Fig. 2. The time-expanded graph of the physical topology of nodes is superimposed with edges to build a space-time network.

structure derived above is purposefully designed to capture the node topology dynamics and model V2V communication opportunities that arise over the time horizon $\mathcal{T}$.

In what follows, a mathematical programming model is constructed to enable an optimized exchange of files. The primary objective of this work is to achieve an even distribution of packets across the group of cooperating vehicles. To do so, let variable $x_{i j}$ be the flow variable across edges in $\mathcal{E}_{1} \cup \mathcal{E}_{2}$ and $x_{i j}^{k}$ be the flow variable for edges $\mathcal{E}_{3}$. Since node replication occurs every $\tau$ units of time, the capacity of all transmission links is set to unity, $u_{i j}=1,(i \mapsto j) \in \mathcal{E}_{1}$ while for edges in $\mathcal{E}_{2}$ the capacity is set to $u_{i j}=F,(i \mapsto j) \in \mathcal{E}_{2}$ Finally, the capacity of edges in $\mathcal{E}_{3}$ is set to unity, $u_{i j}^{k}=1,(i \mapsto j)^{k} \in \mathcal{E}_{3}$.

The cost of traversing a link in $\mathcal{E}_{1}$ is set to unity, $c_{i j}=1$ to account for the number of packet exchanges made. Evidently, an objective function that minimizes the cost on these links will inevitably lead to the least number of file transfers made. Moreover, the cost on links in $\mathcal{E}_{2}$ is neglected hereafter since it does not affect the design considerations of this work. On the contrary, the cost on the $\mathcal{E}_{3}$ edges is chosen in such a way to impose a load balancing effect that will evenly distribute packets across the available nodes. This cost is defined as follows:

$$
c_{i j}^{k}=q \times k, \forall k, k=\{1, \ldots, F\}
$$

where $q$ is an arbitrarily large value incurred by allowing a flow to traverse edge $(i \mapsto j)^{k} \in \mathcal{E}_{3}$. With an objective func- tion that minimizes the cost over these edges, the minimum load variance across all VSs is achieved. Since collecting an increasing number of packets incurs a proportionally higher cost, an optimal solution will only send flows through the cheapest edges and thus achieve load balancing.

The supply of all nodes $i=\left(p_{m}, 1\right), \forall m \in \mathcal{M}$ is $b(i)=F_{m}$, the demand for SS is set to $F$ and for all other nodes, $b(i)=0$.

To find the optimal reallocation policy, the following minimization problem is formulated:

$$
\begin{aligned}
\text { (P1) } \min & \sum_{(i \mapsto j) \in \mathcal{E}_{1}} c_{i j} x_{i j}+\sum_{(i \mapsto j)^{k} \in \mathcal{E}_{3}} c_{i j}^{k} x_{i j}^{k} \\
\text { s.t. } & \sum_{j:(i \mapsto j) \in \mathcal{E}_{1}} x_{i j} \leq 1, \forall i \in V_{p}, t=1, \ldots, T-1 \\
& \sum_{j:(j \mapsto i) \in \mathcal{E}_{1}} x_{j i} \leq 1, \forall i \in V_{p}, t=2, \ldots, T \\
& \sum_{j:(i \mapsto j) \in \mathcal{E}_{1} \cup \mathcal{E}_{2}} x_{i j}-\sum_{j:(j \mapsto i) \in \mathcal{E}_{1} \cup \mathcal{E}_{2}} x_{j i}=b(i), \forall i \in V_{1} \\
& \sum_{j:(i \mapsto j)^{k} \in \mathcal{E}_{3}} \sum_{k=1}^{F} x_{i j}^{k}-\sum_{j:(j \mapsto i) \in \mathcal{E}_{1}} x_{j i}=b(i), \forall i \in V_{2} \\
& \sum_{j:(j \mapsto i)^{k} \in \mathcal{E}_{3}} \sum_{k=1}^{F} x_{j i}^{k}=b(i), i=\mathrm{SS} \\
& 0 \leq x_{i j} \leq u_{i j},(i \mapsto j) \in \mathcal{E}_{1} \cup \mathcal{E}_{2}, x_{i j} \in \mathbb{Z} \\
& x_{i j}^{k} \in\{0,1\},(i \mapsto j)^{k} \in \mathcal{E}_{3}
\end{aligned}
$$

where $V_{1}=V_{p} \backslash\left(p_{m}, T\right), \forall m \in \mathcal{M}$ and $V_{2}=$ $\left(p_{m}, T\right), \forall m \in \mathcal{M}$. Equations (3) and (4) are the outdegree and indegree constraints that restrict each station to transmit/receive data from a single other node at any one time. Constraints (5-7) are the flow balancing constraints for the network flow problem and equations (8-9) define the flow variables.

Theorem 1. The constraint matrix in $(P 1)$ is totally unimodular and thus the linear programming relaxation of $(P 1)$ guarantees integer optimal solutions.

Proof. Firstly, notice that excluding constraint equations (3) and (4) results in a constraint matrix identical to that of the minimum cost flow problem that enjoys the integrality principle [13]. Hence, given integer supply/demand values and integral lower/upper bounds on the flow variables (as is the case here), integer optimal solutions are guaranteed.

With the introduction of equations (3) and (4) the following additions are made to the constraint matrix:

- For every node in $V_{p}$ one additional row is added to the node-arc incidence matrix. On that row, all entries of the matrix associated with outdegree links from each node are set to 1 .

- For every node in $V_{p}$ one additional row is added to the node-arc incidence matrix and all entries of the matrix associated with indegree links to each node are set to 1 . In effect, each column in the constraint matrix corresponding to edges in $\mathcal{E}_{1}$ is amended with entries of unity value. Since, this is the only addition made to the constraint matrix, it can be shown that this columns can be rearranged in such a way that 


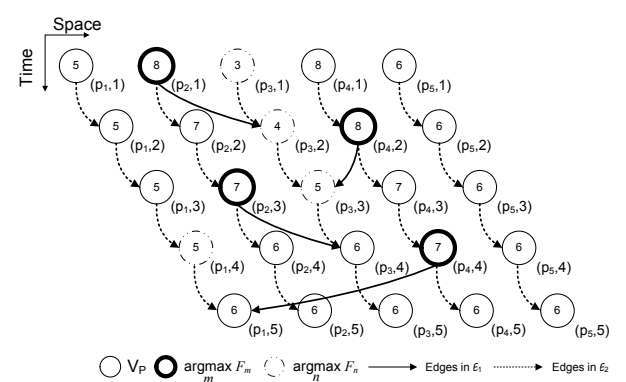

(a)

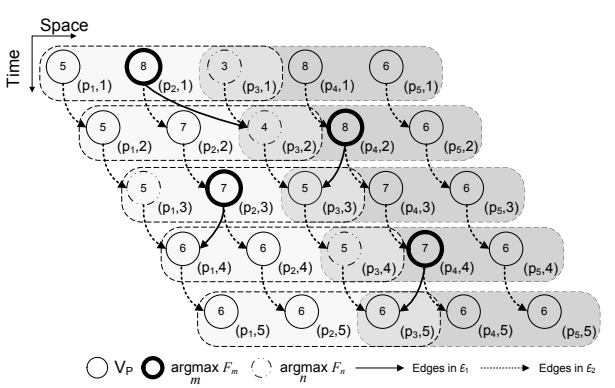

(b)

Fig. 3. Example schematics of the multiplicity of reallocations that suffice. In plot (b) the two regions are represented by distinct shades of gray.

the determinant of each square submatrix is 0 or \pm 1 and thus the constraint matrix is totally unimodular. As shown in [14], totally unimodular matrices adhere to the integrality property.

It should be emphasized here that for the case where each VS is in direct contact with every other node in its group, the problem solution is trivial: At each time step $t \in \mathcal{T}$, node $m$ having the largest buffered load (i.e., $\arg \max _{m}\left\{F_{m}\right\}$ ) forwards a single packet to node $n$ with the lowest buffered load (i.e., $\arg \min _{n}\left\{F_{n}\right\}$ ) until the best possible load dissemination is achieved or when all reallocation moves in horizon $\mathcal{T}$ have been filled. Obviously, this is the best possible solution since every path is one hop long. In the alternative case where the average path length is longer than one, the combinatorial nature of the problem surfaces. Illustratively, consider the example depicted in Figure 3 with five nodes exchanging files in succession. Figure $3 a$ depicts the case where every node can communicate directly with every other node, while Figure $3 \mathrm{~b}$ presents a case where two communication regions are formed due, for example, to the limited communication range of nodes. In the latter case, node 3 is a member of both regions.

As discussed above, when all paths are one hop long, load balancing can be achieved by moving files from nodes having the largest buffers to nodes having the shortest ones (in occupancy) as shown in Figure 3a; with ties broken arbitrarily. In contrast, when communication regions are considered (as shown in Fig. 3), numerous alternative forwarding decisions become viable with inherently varying communication costs. If, for example, instead of using link $\left(p_{2}, 3\right) \mapsto\left(p_{1}, 4\right)$, link $\left(p_{2}, 3\right) \mapsto\left(p_{3}, 4\right)$ was chosen in forwarding files on the graph depicted in Figure $3 b$, then due to the limited communication of nodes, a much greater number of transfers would be needed

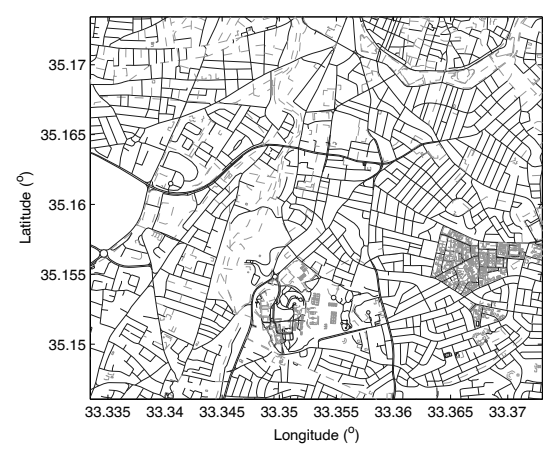

Fig. 4. Simulation area under consideration. The area around the largest part of the city of Nicosia, Cyprus is used.

to achieve the same level of load balancing.

Obviously, the latter scenario is anticipated in most realistic settings and thus (P1) is employed to find optimal file transfers.

\section{PERFormance Evaluation}

In this section, the performance of ExTra is evaluated against traditional forwarding (i.e., when no prior file exchange is conducted). In the evaluation, the area around the largest part of the city of Nicosia in Cyprus is considered, as shown in Figure 4. On independent runs, 1) a single route is selected from the map, 2) the route is populated with vehicles, and 3) a single random source node is selected among the VSs. We assumed that at the end of each route there is an available AP for data delivery. Subsequently, a space-time network $G$ is created based on the observed neighborhood of the source node. Lastly, problem (P1) is solved by a source node to derive an optimized file transfer strategy.

For the results presented below, an average node density of 0.05 vehicles $/ \mathrm{m}$ is assumed. Moreover, to estimate future vehicle positions (necessary to building network $G$ ), Gipp's carfollowing model was employed, using the following standard modelling parameters: the maximum speed was set to $13 \mathrm{~m} / \mathrm{s}$, vehicle acceleration was set to $1.7 \mathrm{~m} / \mathrm{s}^{2}$, actual deceleration was set to $3 \mathrm{~m} / \mathrm{s}^{2}$, while the deceleration estimated by following drivers was set to $2.5 \mathrm{~m} / \mathrm{s}^{2}$. The vehicle size was set to $5 \mathrm{~m}$ and the driver reaction time was assumed to be $1 \mathrm{sec}$. In regards to the data traffic, $F_{m}, \forall m \in \mathcal{M}$ is assumed to be uniformly distributed in the interval $\{0,20\}$ files, the transmission rate is $R=1 \mathrm{Mbps}$ and the transmission duration is $\tau=1 \mathrm{sec}$. These parameters are also used for the results in section IV.

Figure 5 compares the initial loading variance of the $F_{m}, \forall m \in \mathcal{M}$ values to the resulting load variance achieved after every file transfer is made as suggested by the optimal solution to $(\mathrm{P} 1)$. The four plots in the figure contain results for increasing windows of time horizon $\mathcal{T}: T=1 \mathrm{sec}$ for Fig. 5a, $T=5 \mathrm{sec}$ for Fig. 5b, $T=10 \mathrm{sec}$ for Fig. $5 \mathrm{c}$, and $T=15 \mathrm{sec}$ for Fig. 5 d.

Evidently, the availability of extended time windows (i.e., increasing $T$ ) before accessing the infrastructure allows for an increasing number of exchange opportunities and thus bigger improvements in load balancing can be realized. As illustrated in the plots of Fig. 5, there is only a marginal improvement for $T=1$ by the proposed strategy (Fig. 5a), while there is a clear improvement for a mere $T=5 \mathrm{sec}$ time window (Fig. $5 \mathrm{~b}$ ) 


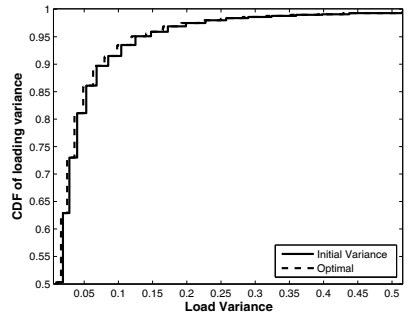

(a)

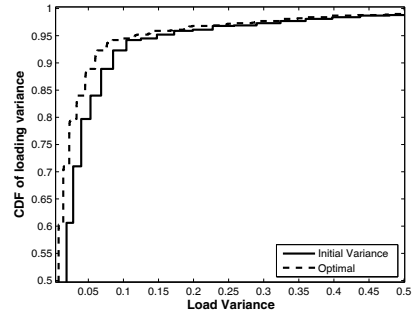

(b)

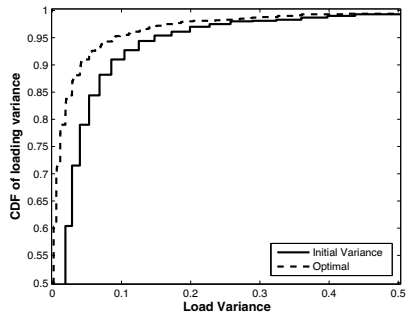

(c)

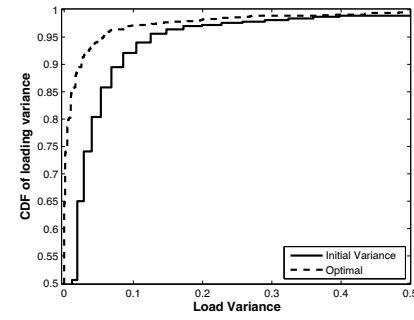

(d)

Fig. 5. Performance comparisons for varying values of time window $\mathcal{T}$ : (a) $T=1 \mathrm{sec}$, (b) $T=5 \mathrm{sec}$, (c) $T=10 \mathrm{sec}$, and (d) $T=15 \mathrm{sec}$.

and a substantial gain when a time window of $T=10 \mathrm{sec}$ and $T=15 \mathrm{sec}$ is available (Fig. 5c, and Fig. 5d, respectively).

\section{Modelling Considerations}

The derivations in Section II and the discussions that followed in Section III concentrated on a single instance of the problem where a source node decides on its file transfer policy, based solely on 1) its current view of available neighboring nodes and 2) information exchanged between these nodes in regards to the volume of buffered data traffic of each node.

Clearly, both the volume of data traffic generated and the structure of the node topology (as seen by the source node) change dynamically. Moreover, due to unanticipated changes in a vehicle's path, VSs may defer data delivery until an alternative access point becomes available. On top of that, the coexistence of multiple source nodes with uncoordinated file transfer policies might result in conflicting and unnecessary file exchanges. In what follows, each of the three issues identified here, are being addressed to improve the robustness of the proposed solution.

\section{A. Iterative Optimization}

In constructing an optimized file transfer policy, a source node invokes the following steps:

1) Retrieves information on the volume of traffic buffered at each neighboring vehicular station.

2) Builds space-time network $G$ based on current and forecasted node mobility.

3) Solves (P1) to obtain an optimized file transfer strategy.

As previously mentioned, since new content may be generated dynamically and uncontrolled mobility incidents may cause a deviation from anticipated paths, decisions made at one instance may become outdated (or even obsolete) over time. Consequently, a source node needs to iteratively update its file transfer policy by re-computing (P1) periodically or when deemed necessary. Evidently, problem (P1) can be efficiently solved in practice (as shown by Theorem 1) and thus an iterative solution becomes viable. Algorithm 1 details the steps taken to maintain the most updated strategy.

Figure 6 plots the observed execution times of solving problem (P1) for various lengths of time horizon $\mathcal{T}$. Looking into the cumulative distribution of the recorded times, more than $90 \%$ of the test runs can be solved in less than $0.2 \mathrm{sec}$ on a standard general purpose machine. It is thus evident that (P1) can be solved at run-time to compensate for dynamic

\begin{tabular}{l}
\hline Algorithm 1 Iterative Optimization Algorithm \\
\hline 1: repeat \\
2: $\quad$ Communicate buffered data traffic information to the \\
s: $\quad$ Build space-time network $G$ and solve (P1). \\
4: $\quad$ Update and execute the computed file transfer strategy. \\
5: until Data delivery completed \\
\hline
\end{tabular}

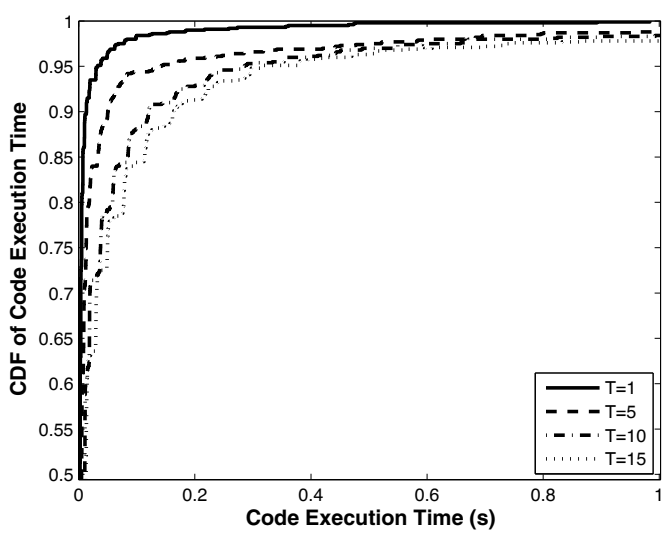

Fig. 6. Matlab code execution time of a standard general purpose machine.

networking changes. Moreover, it is clear that for increasing values of $T$, the size of $G$ increases and thus computational time becomes proportionally higher, as shown in the figure.

\section{B. Access Point Multiplicity}

Inevitably, each vehicle participating in ExTra will deviate from the group to follow its personal mobility objectives. For that reason, the time horizon should be kept small enough to avoid the transfer of files to nodes that will deviate from the anticipated path. A possible time-span for $T$ could be for example the time required for nodes to reach the next road junction. Even so, there could be cases where due to the availability of journey planners, highly reliable estimates of future vehicle paths can be considered.

In those cases, a multiplicity of access points could be incorporated in the optimization process. Illustratively, Figure 7 depicts one realization of the space-time network $G$ where VSs follow two distinct paths that approach two adjacent APs.

As before, a source node should aim at maximizing the distribution of files across the available nodes. However, with the introduction of additional APs the following amendments 


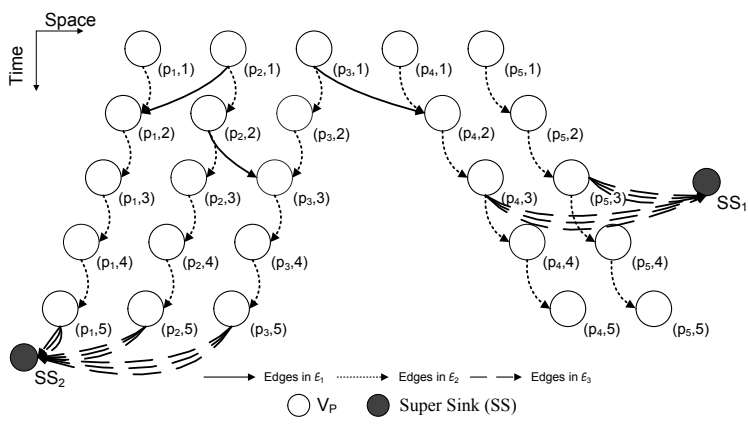

Fig. 7. Space-time network that incorporates the numerous access points that may become available within the forecasted time window $\mathcal{T}$.

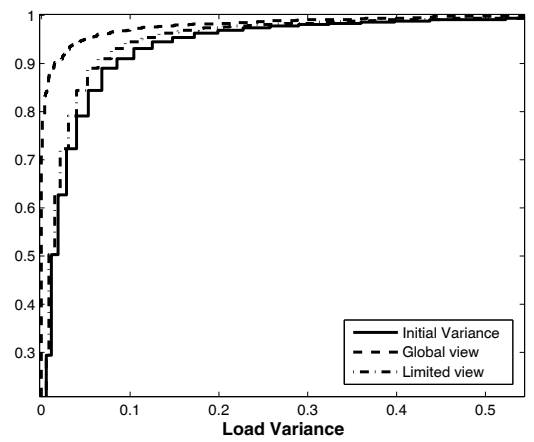

Fig. 8. Performance of global optimal and local optimal solutions as compared to the initial load variance across the cooperating vehicular stations.

should be made to the networking model described in Section II:

- Instead of a single SS node, the set $\mathcal{S}$ is used for the collection of sink nodes.

- The definition of $\mathcal{E}_{3}$ is updated as follows: An edge $(i \mapsto$ $j)^{k} \in \mathcal{E}_{3}$ carries the flow $k$ between VS replica $i=$ $\left(p_{i}, T\right)$ to sink node $j \in \mathcal{S}$.

- Constraint equation (7) is updated as follows: $\sum_{(i \mapsto j)^{k} \in \mathcal{E}_{3}} x_{i j}^{k}=F$.

Noticeably, the only change imposed on (P1) is the flow balancing constraint that is altered to reflect on the demand of flows that needs to be collected not by a single SS but by the set of available sink nodes in $\mathcal{S}$. Computationally, total unimodularity of the constraint matrix is preserved and thus the complexity of (P1) does not change.

\section{Source node multiplicity}

In all previous discussions, it was assumed that a single source node was active among the available VSs. It was further assumed that this node had a complete picture of the underlying topology. Both assumptions are relaxed here. Clearly, at any point in time, there can be a number of active source nodes. Moreover, each of these source nodes may only have partial view of the network topology due for example to the limited communication range and the large dimensioning of the topology. In effect, each file exchange strategy will be limited to the confined view obtained by each source node.

Figure 8 illustrates the achieved load balancing levels when two source nodes are present within the set $\mathcal{M}$ of VSs with a time window of $T=10 \mathrm{sec}$. In the first case, both source nodes have global view of the topology, while in the second case, the group of vehicles is partitioned into two equally large set of nodes that are found closer to the sources.

As anticipated, the limited view of the topology greatly penalizes performance. Evidently, to improve on this bound, some form of communication should take place to guide the exchange of files. Designing such a communication protocol is left as future work.

\section{CONCLUSIONS}

Due to the limited coverage of hotspots and the heighten level of mobility by vehicular stations, communication links are destined to be short-lived and thus of limited use. To maximize the forwarding opportunities, this work proposes the use of vehicle-to-vehicle communication in an effort to disseminate data fragments across neighboring VSs that are willing to assist in the offloading process.

A systematic mathematical framework is derived to model this problem and optimized forwarding strategies are derived by formulating and solving the reallocation programming problem. Finally, a detailed analysis of the performance of the proposed programming formulation is conducted, demonstrating the applicability of ExTra.

\section{ACKNOWLEDGMENTS}

This work was supported by the Cyprus Research Promotion Foundation's Framework Programme for Research, Technological Development and Innovation (DESMI 2008), co-funded by the Republic of Cyprus and the European Regional Development Fund, and specifically under Grant Project New Infrastructure/ Strategic/0308/26).

\section{REFERENCES}

[1] B. Fleming, "Smarter Cars: Incredible Infotainment, Wireless Device Charging, Satellite-Based Road Taxes, and Better EV Batteries", IEEE Vehicular Technology Magazine, 8(2):5-13, 2013.

[2] ERCIM, "Special Theme: Intelligent Cars", ECRIM News, No. 94, Page(s):4 - 25, July 2013.

[3] European Commision, Standardisation Mandate addressed to CEN, CENELEC and ETSI in the field of information and communication technologies to support the interoperability of cooperative systems for intelligent transportation in the European community, Mandate 453, Oct. 2009.

[4] K. Dar, M. Bakhouya, J. Gaber, M. Wack and P. Lorenz, "Wireless communication technologies for ITS application", IEEE Communications Magazine, 48(5):156-162, 2010.

[5] ETSI, "Intelligent Transport Systems (ITS); Cooperative ITS (C-ITS); Release 1", Technical Specification 101607 V1.1.1, 2013.

[6] International Transport Forum, "Transport Outlook 2012, Seamless Transport for Greener Growth", OECD/ITF, 2012, www.internationaltransportforum.org.

[7] EC Press Release, "Mobile communications: Fresh 50 million EU research grants in 2013 to develop '5G' technology", IP/13/159, Feb. 2013.

[8] Cisco, "Visual Networking Index (VNI) Global Mobile Data Traffic Forecast for 2011 to 2016", Cisco VNI Forecast, Feb. 2013.

[9] A. Petrescu, et. al., "Scenarios and Requirements for IP in Intelligent Transportation Systems", Internet-Draft v.02, Mar. 2013, http://tools.ietf.org/html/draft-petrescu-its-scenarios-reqs-02\#section-3.1.

[10] A. Garca, et al., "D2.1 Use Cases and Requirements", Mobile Opportunistic Traffic Offloading Project, Feb. 2013, http://www.fp7-moto.eu/.

[11] J. Eriksson, H. Balakrishnan and S. Madden, "Cabernet: Vehicular Content Delivery Using WiFi”, ACM International Conference on mobile computing and networking, Page(s):199 - 210, Sept. 2008.

[12] ETSI, "Intelligent Transport Systems (ITS); Vehicular Communications; Basic Set of Applications; Part 2: Specification of Cooperative Awareness Basic Service", Technical Specification 102 637-2 V1.2.1, 2011.

[13] R. Ahuja, T. Magnanti and J. Orlin, Network Flows: Theory, Algorithms and Applications, Prentice Hall, 1993.

[14] A. Schrijver, Theory of Linear and Integer Programming, Wiley Press, April 1998. 